\title{
Un mejor método electrocardiográfico para medir la hipertrofia ventricular izquierda y predecir eventos cardiovasculares
}

Prognostic Value of a New Electrocardiographic Method for Diagnosis of Left Ventricular Hypertrophy in Essential Hypertension. Verdecchia P, Schillaci, et al. J Am Coll Cardiol 1998, 3138390

\author{
Objetivo \\ Evaluar el valor pronóstico de un nuevo método electrocardiográfico \\ (puntaje de Perugia) (ECG), para medir la hipertrofia ventricular iz- \\ quierda (HVI) y compararlo con cinco métodos estándares.
}

\section{Diseño}

Estudio prospectivo de cohortes, con seguimiento de hasta diez años (media 3,3).

\section{Lugar}

Tres hospitales de Umbria, Italia.

\section{Pacientes}

Se incluyeron 1784 pacientes con hipertensión esencial en el contexto de un estudio para el monitoreo del presión arterial (PIUMA), sin evidencia de insuficiencia cardiaca o hipertensión arterial secundaria.

\section{Evaluación de los factores pronósticos}

Se realizó a todos los participantes en forma ciega, un ECG y un Ecocardiograma. Se evaluó en cada paciente los siguientes métodos ECG para diagnosticar HVI:

Criterio de Sokolow : la suma de la onda S en V1 con la R de V5 o V6 35 mv. Puntaje de Romhilt Estes: onda R en V5 ó V6 de 30 mm., ó S en V1 o V2 > de $30 \mathrm{~mm}$., suma 3 puntos; deflexión negativa de la onda P en V1> a 0,04 seg., suma tres puntos; sobrecarga de ventrículo izquierdo, suma tres puntos; desviación del eje a la izquierda, suma dos puntos. Se considera positivo valores superiores a 5 puntos.

Voltaje de Cornell: suma de las ondas S de V3 y R de AVL $>28 \mathrm{mv}$. en hombres y 20 en mujeres.

Criterios de Framingham: requiere la presencia de sobrecarga ventricular izquierda con uno de los siguientes criterios; criterio de Sokolow, o La suma de las ondas R en DI y $\mathrm{S}$ en DIII > a $25 \mathrm{mv}$.,ó la onda S en las precordiales derechas ó $\mathrm{R}$ en las precordiales izquierdas mayor a $25 \mathrm{mv}$. Sobrecarga ventricular izquierda:depresión asimétrica de la onda Ten las precordiales izquierdas.
Puntaje de Perugia: requiere la positividad de uno de los siguientes tres criterios. Voltaje de Cornell, presencia de sobrecarga ventricular izquierda ó un puntaje de Romhilt Estes mayor ó igual cinco.

\section{Medición de resultados principales}

Se midieron los eventos cardiovasculares fatales y no fatales por médicos que estaban ciegos a los otros datos de los pacientes. Mediante un análisis multivariado se calculó la relación entre la enfermedad cardiovascular, la prevalencia de HVI, y el poder de predicción de cada uno de los métodos diagnósticos de HVI. Las variables incluidas en el ajuste fueron: edad, diabetes, tabaquismo, enfermedad cardiovascular previa, e hipertensión.

\section{Resultados Principales}

Utilizando el puntaje de Perugia, un $17,8 \%$ de los pacientes hipertensos tenían HVI comparado con los otros métodos; Cornell $(9,1 \%)$, Framingham (3,9\%), puntaje de Romhilt Estes $(5,2 \%)$, sobrecarga $(6,4 \%)$ y el índice de Sokolow $(13,1 \%)$. En el seguimiento se documentaron 159 eventos cardiovasculares. La tasa de eventos cardiovasculares fue mayor para aquellos pacientes con HVI detectada con todos los métodos excepto con el índice de Sokolow. El riesgo relativo ajustado para padecer eventos de los distintos puntajes fue: Cornell: 1.34 (IC 95\% 0.86 2.08, p NS); Framingham: 1.91 (IC 95\% 1.13-3.23, p 0.05); RomhiltEstes: 2.63 (IC 95\% 1.69-4.10); Sobrecarga: 2.11 (IC95\% 1.40-3.20); Sokolow: 1.19 (IC95\% 0.77-1.83); Puntaje Perugia: 2.04 (1.47-2.80). El índice de Perugia demostró ser el método con más alto valor pronóstico $(15,6 \%)$, comparado con el Framingham, Romhilt-Estes y sobrecarga con $3 \%, 7,4 \%$ y $6,8 \%$ respectivamente.

\section{Conclusiones}

En base al riesgo de eventos, el índice de Perugia mostró el mayor rendimiento para el diagnóstico de HVI comparado con los métodos clásicos. El ECG conserva un importante rol en la estratificación del riesgo cardiovascular en pacientes hipertensos.

Fuente de financiamiento: Associazione Umbria Cuore e Ipertensione, Perugia, Italia.

\section{COMENTARIO}

La HVI en la hipertensión es un factor de riesgo independiente para muerte súbita, infarto de miocardio y accidente cerebrovascular ${ }^{1-2}$ Existe evidencia de estudios observacionales que demuestra que la regresión de la HVI, que se observa con el tratamiento no farmacológico y más aun con el farmacológico de la hipertensión, se asocia con una disminución de los eventos cardiovasculares ${ }^{3}$; pero lo que aún no se sabe es si este efecto es independiente del descenso de la presión. Previamente a la existencia de drogas para el tratamiento de la hipertensión, cerca del $40 \%$ de los hipertensos tenían como causa de muerte la miocardiopatía hipertensiva. Por lo tanto es muy importante en un paciente hipertenso tanto en el inicio como en el sequimiento, documentar la presencia de HVI.

EL ECG es el método de rutina para la detección de HVI en pacientes con hipertensión, dado su fácil empleo, alta disponibilidad y bajo costo. Este es el primer estudio que permite comparar entre sí los distintos métodos basados en el ECG para medir HVI, y valorar en forma independiente la capacidad de cada uno para predecir eventos cardiovasculares. La muestra del estudio es representativa de la población de hipertensos esenciales que normalmente se ve en el consultorio, ya que los pacientes eran enviados por médicos de atención primaria, e incluyó un $68 \%$ de hipertensos no conocidos previamente. La tasa de eventos del estudio es muy similar a la de los pacientes con hipertensión esencial en la población general. Esto elimina uno de los sesgos clásicos de los estudios que valoran tests

*Ver Glosario

\section{Dr. Augusto Granel}

Unidad de Medicina Familiar y Preventiva. Hospital Italiano de Buenos Aires.

1. Koren MJ, Devereux RB, Casale PN. Relation of left ventricular mass and geometry to morbidity and mortality in uncommplicated essential hypertension. Ann Intern Med 1991; 114: 345-352 2. Haidar AN, Larson MG. Increased left ventricular mass and hypertension are associated with increased risk for sudden death. J Am Coll Cardiol 1998; 32 (9): 1454-9

3. Levy D, Salomon M, D' Agostino PB. Prognostic implications of baseline electrocardiogram features and their serial changes in subjets with Left ventricular hypertrophy. Circulation 1994; 90: $1786-1793$

diagnósticos que es la selección de una muestra muy sana o muy enferma. Un aspecto interesante es la escasa capacidad tanto de detección como de predicción del criterio de Sokolow, a pesar de que no solamente es el más utilizado si no también el más difundido por los textos habituales. Del estudio se desprende claramente que no debe utilizarse un solo criterio diagnóstico para HVI si no que deben considerarse y sumarse distintos en un puntaje, del cual el Puntaje de Perugia parece ser el mejor predictor. Si bien los pacientes con HVI diagnosticada por el método de Perugia tienen un riesgo relativo de sufrir eventos cardiovasculares menor a los detectados por el de Romhilt-Estes, su rendimiento clínico global es mejor, ya que el primer método detecta más pacientes. A simple vista parece complicado hacerlo, pero en la práctica no se tarda mas de cinco minutos, sobre todo si consideramos que los pacientes hipertensos deben tener un ECG siempre inicialmente y luego uno cada uno a dos años. Debe pensarse que esta simple medición con un sencillo método, tiene gran implicancia para el pronóstico de los pacientes.

Otro aspecto que sería interesante investigar por los autores, es comparar la capacidad predictiva de eventos cardiovasculares del ecocardiograma, utilizado en el estudio como test de referencia, con los métodos basados en el ECG. Si la diferencia fuera muy marcada, el próximo paso sería determinar su costo-efectividad. 